\title{
Kann es ein Unbewusstes geben?
}

Vera Saller (Zürich)

Zusammenfassung: Die Arbeit vertritt die These, dass das Freud'sche Konzept des Triebes auch im Rahmen einer modernen Theorie des Denkens sinnvoll ist. Nach der Feststellung, dass mit dem von Peter Fonagy und Mary Target formulierten Entwicklungsziel Mentalisierung das Rekurrieren auf die Freud'sche Triebtheorie mehr und mehr in den Hintergrund gedrängt wird, versucht die Autorin anhand einer Diskussion zwischen analytischer Philosophie und Psychoanalyse, die in den letzten Jahrzehnten im englischen Sprachraum geführt worden ist, den theoretischen Stellenwert der Triebtheorie herauszuarbeiten. Die Gemeinsamkeit von Mentalisierungstheorie und philosophischer Bedeutungstheorie im Sinne Donald Davidsons ist, dass beide Theorierichtungen die zentrale Rolle der geteilten Aufmerksamkeit auf die Welt, und das Konzept der Triangulation anerkennen und betonen.

Schlüsselwörter: (3-10) Mentalisierung, Theory of Mind, Triangulation, Dualismus. Intentionalität, Externalistische Bedeutungstheorie, Dualismus, Finale Kausalität.

Das Konzept der Mentalisierung verbindet die Psychoanalyse mit Wissen von anderen Disziplinen, insbesondere der Philosophie, Kognitionswissenschaft und Bindungstheorie. Peter Fonagy und Mary Target bringen mit dem Begriff der Mentalisierung neu in die Debatte ein, dass die Entwicklung des Kleinkindes daraufhin untersucht und bewertet wird, inwiefern es den Kindern möglich ist, den andern als eine Person mit eigenen Wünschen und Zielen anzuerkennen. In den diesbezüglichen Forschungen wird darüber debattiert, ob das Kind über eine Theory of Mind verfüge. Angesichts der starken Orientierung der Autoren an den Ergebnissen psychologischer und kognitionswissenschaftlicher Beforschung von Kleinkindern stellt sich allerdings die Frage, ob die Psychoanalyse sich mit dieser Einbindung in eine allgemeine Wissenschaft vom Menschen nicht zu sehr von ihren theoretischen Wurzeln entfernt. Insbesondere scheint mir problematisch, dass eine ideale, normative Entwicklungslinie vorgezeichnet wird und die Abweichungen davon nur noch als Defizite charakterisiert werden. Dagegen ist das psychoanalyti- 
sche Vorgehen bestrebt, vordergründig als sinnlos und defizitär erlebten Störungen mit Bezug auf tiefere Triebkräfte einen bis dahin unerkannten Sinn zuzuordnen. In Frage steht deshalb, wie weit es ratsam sei, mit der pragmatischeren Theorie der Mentalisierung wesentliche Teile der Psychoanalyse aufzugeben.

Inwiefern die Konzepte Unbewusstes, Primärprozess und Trieb in einer modernen Theorie des Denkens Platz haben können, ist der Gegenstand einer verwandten, aber nicht identischen Theoriedebatte, die ich vorstellen werde. Die Debatte zwischen analytischer Philosophie und Psychonalyse könnte in einer bestimmten Hinsicht als das genaue Gegenstück zu den theoretischen Bemühungen der Autorengruppe um Fonagy gesehen werden: Geht es letzterer darum, dasWissen vom normalen Denken in die psychoanalytische Theoriedebatte einzubringen, wird dort gefragt, ob in den Theorien, die Denken beschreiben, wie es im Normalfall funktioniert, auch irrationale Aspekte eingefügt und verstehbar gemacht werden können. Mit anderen Worten: ob im Rahmen einer externalistischen Bedeutungstheorie das Unbewusste, wie Freud es konzipiert, überhaupt denkbar sei. Ein wichtiges Thema in der heutigen Philosophie des Geistes ist die Überwindung des Leib-Seele-Dualismus. Ich werde dies anhand einer Debatte zum Thema Intentionalität zeigen. Danach werde ich die Ansicht vertreten, dass gerade der Freud'sche Triebbegriff, wie er in den Drei Abhandlungen zur Sexualtheorie eingeführt wird, als Grundbegriff einer embodied theory of mind dienen könnte, die sowohl Rationales wie Irrationales umfasst.

\section{Mentalisierung}

Dem ausgesprochen synthetischen Ansatz, den uns die Autoren in Affect Regulation, Mentalization and the Development of the Self präsentieren, gelingt es, eine Unmenge theoretischer und erfahrungswissenschaftlich erhobener Daten und Theorieansätze zu einem theoretischen Bogen zu verarbeiten, der die zunehmend gelingende Affektregulierung des Säuglings beschreibt und diese als Voraussetzung dessen begreift, was das Kleinkind befähigt, den anderen als Menschen mit einer eigenen Perspektive wahrzunehmen. Was die Bedeutung der Affektspiegelung durch die das Baby betreuende Person betrifft, können die Autoren auf eine breite und lange Theorietradition zurückblicken ${ }^{1}$. Bei der Zusammenführung der Theorielinien Ödipalität (z. B. nach Britton et al. 1989) und der Entwicklung dessen, was wir zeitgenössische Psychoanalytiker als psychische Realität zu bezeichnen gewohnt sind, zeigt sich indessen die Kraft und Originalität ihrer Synthese. Dabei ist anzumerken, dass die Ergebnisse der Baby-Beforschung akribisch auf implizite Vorannahmen hin untersucht werden. Fonagy et al. distanzieren sich z. B. von jenen 
Theorie-Ansätzen, die eine angeborene Intersubjektivität postulieren und gemäss der Kleinkinder die Fähigkeit, den anderen als Menschen mit Intentionen zu verstehen, gar nicht erlernen müssten (vgl. ebd. S. 210 ff.). Genau dieser Lernprozess ist es nämlich, der Fonagy et al. interessiert; er steht im Zentrum der Untersuchung des Autorenkollektivs. Die differenzierte Analyse der Entwicklungsprozesse gibt den Autoren Anlass, gewisse Krankheitsbilder von Erwachsenen als fehlgeleitete, respektive stecken gebliebene Lernvorgänge in den entsprechenden Phasen wieder zu erkennen. Fonagy et al. beanspruchen, mit dem Konzept der erst allmählich entstehenden Subjektivität auch auf der Ebene der Philosophie die dualistischen, cartesianischen Annahmen überwunden zu haben. Dieser Anspruch wird in den unten zur Besprechung gelangenden philosophischen Ansätzen eine wichtige Rolle spielen. Dabei wird Cartesianismus gleichgesetzt mit der als solipsistisch gebrandmarkten, aber als richtig erlebten Annahme, dass der Grundstein zum Erkennen in der Wahrnehmung der Realität durch den Einzelnen liege, und dass subjektive Erlebnisweisen dem Einzelnen ohne vorheriges Erlernen zugänglich seien.

Mit der Ablehnung der bis dahin auch in weiten Teilen der Psychoanalyse intuitiv und implizit geltenden Vorannahme der angeborenen Subjektivität gelingt es Fonagy et al., eine weitere Theorielinie einzubeziehen, die bis dahin in der Psychoanalyse gänzlich ignoriert wurde. Es geht um die aus der amerikanischangelsächsischen Philosophie-Tradition erwachsene Mischung von Philosophie des Geistes und Kognitionswissenschaft, die den Erwerb einer Theory of Mind untersucht, d.h. die zu verstehen sucht, wie das Kind dazu kommt, den anderen als denkendes Wesen mit Absichten und Wünschen zu erleben.

\section{Was unterscheidet den Menschen vom Tier?}

Ich werde nun auf einen der Forscher eingehen, den die Autoren als Beleg für die Mentalisierung angeben, den Biologen und Anthropologen Michael Tomasello. In seinem Buch The Cultural Origin of Human Cognition (1990) zeigt er, dass die Grenzen zwischen Mensch- und Tierreich sehr genau gezogen werden können, trotz in jüngerer Zeit vermehrt auftauchender Indizien und Forschungsbefunden, die besagen, dass Tierkinder höherer Gattungen viel mehr von ihren Eltern beigebracht bekommen, als wir bis dahin annahmen. So entwickeln sich höhere Primaten- und Menschenbabys bezüglich Nähe und Nachahmungstrieb zur mütterlichen Pflegeperson ähnlich bis zu dem Punkt, wo Mütter und ca. 9 Monate alte Kinder anfangen, aus der sie umgebenden Umwelt durch Zeigen und gegenseitiges Beobachten der Blickrichtung Objekte zum Thema eines Austausches zu definieren. Dieses gemeinsame Erkunden derWelt spielt natürlich beim Spracherwerb, der 
damit einsetzt, eine ganz entscheidende Rolle. Ich zitiere eine Stelle, in der das dyadische Verhalten eines Proto-Austausches von Gefühlen, Lauten und Berührungen des Babys mit dem ab dem 9. Monat einsetzenden triadischen Verhalten verglichen wird:

«Six-month infants interact dyadically with objects, grasping and manipulating them, and they interact dyadically with other people, expressing emotions back and forth in a turn-taking sequence. If people are around when they are manipulating objects, they mostly ignore them. If objects are around when they are interacting with people, they mostly ignore them. But at around nine to twelve month of age a new set of behaviors begins to emerge that are not dyadic, like these early behaviors, but are triadic in the sense that they involve a coordination of their interactions with objects and people, resulting in a referential triangle of child, adult, and the object or event to which they share attention» (ebd.62).

Mit der Hervorhebung der Spracherwerbs-Situation und der auf sie folgenden Erweiterung des Horizonts auf Möglichkeitsräume sagt Tomasello im Grunde nichts Neues. Dass die Menschen als Mängelwesen zur Welt kommen, dass sie nicht auf ihren Instinkt vertrauen können und der Platz dieser Sicherheit gebenden automatischen Handlungsanleitung (der Platz der Instinkte) durch menschliche Institutionen eingenommen wird, ist schon von vielen Theoretikern hervorgehoben worden.

Neu ist jedoch, dass Tomasello als Vorbedingung für den Spracherwerb die Triangulierung der Situation nennt - erstaunlicherweise ohne Verweis auf Donald Davidson, der den Begriff der Triangulation als einen Aspekt seiner Bedeutungstheorie innerhalb der Philosophie des Geistes geprägt hat (vgl. unten).

Weiter ist für uns als Psychoanalytiker, die wir gewohnt sind, die menschliche Kleinkindentwicklung im Rahmen psychoanalytischer Vorstellungen zu sehen, aussergewöhnlich, dass Tomasello auf Kognitionstheorie abstellt, d.h. nicht in erster Linie auf die emotionale Bedeutung der Bemutterung abzielt, sondern die Erkenntnisfähigkeit des Menschen überhaupt befragt. Es ist anzufügen, dass Fonagy et al. eine Differenzierung der von ihm gezeichneten Entwicklungslinie der Kleinkindentwicklung erarbeiten, indem sie sich von Tomasellos Vorstellungen abgrenzen (vgl. ebd. 210 ff.). Für mein Vorhaben spielen diese Differenzen indessen 
keine Rolle. Denn beide Autoren beantworten die philosophische Frage danach, wo die Bedeutungen und wo Wissen entstehe in derselben Weise: im Aussen. Sie sind in ihrer Bedeutungslehre, was ich im kommenden Abschnitt näher erläutern möchte, Externalisten.

Ich beende meine Ausführungen zum intrapersonalen Aspekt des Entwicklungsschrittes, den Fonagy et al. mit Mentalisierung bezeichnen, indem ich auf den Freud'schen Triebbegriff zu sprechen komme. Ich möchte die von Freud 1915 in seinem Triebe und Triebschicksale erarbeiteten Begriffe erläutern, die das Wesen der Triebe charakterisieren (vgl. dazu auch Brakel 2009: $88 \mathrm{f}$.).

Beim Triebgeschehen handelt es sich gemäss Freud um einen drängenden Prozess, der den Organismus auf ein Ziel hinstreben lässt. Die Quelle des Triebes ist ein körperlicher Reiz (Spannungszustand); sein Ziel ist die Aufhebung desselben. Das Objekt ermöglicht dem Trieb die Erreichung seines Zieles. Die vier Begriffe, die das Arbeiten des Triebes charakterisieren, sind also: Drang, Quelle, Objekt und Ziel. Der Drang bezeichnet das Prozesshafte eines Vorganges, man könnte auch sagen, den energetischen Beitrag. Die Quelle des Triebes ist der Spannungszustand im Körper sowie das Ziel die Aufhebung desselben ist. Besonders bedeutsam in unserem Zusammenhang sind die folgenden Sätze von Freud:

«Das Objekt des Triebes ist dasjenige, an welchem oder durch welches der Trieb sein Ziel erreichen kann. Es ist das variabelste am Triebe, nicht ursprünglich mit ihm verknüpft, sondern ihm nur infolge seiner Eignung zur Ermöglichung der Befriedigung zugeordnet.» (Freud 1915a/1982a: 86. Hervorhebung des Autors)

Ich meine, es ist das Objekt des Triebes, das in Erinnerungsspuren und Vorstellungen festgehalten wird, das erinnert, phantasiert oder auch verworfen oder verdrängt werden kann. Es sind diese erinnerten Objekte, die durch den inneren Dialog, durch Neubildungen, Metaphorisierungen und Verschiebungen die Sprache im Körper verankern. Das Objekt ist variabel und funktioniert deshalb quasi als Eingangstüre in den primär als geschlossen zu konzipierenden Körperkreislauf mit seinen instinkthaften Abläufen. Mit der Variabilität des Objektes, mit seiner Veränderlichkeit (die indessen überhaupt nicht beliebig ist!) kann sich die Bedeutungsvielfalt der psychischen Welt und der Sprache einschleichen. Sie entsteht durch den intensiven, libidinös besetzten Austausch mit den Erwachsenen, die Erinnerung des Babys an dieselben und seinen Drang, früher einmal als lustvoll erlebte Situationen wieder zu erleben. Das Suchen und 
Verdrängen, Widerauffinden und Vermissen von lustbesetzten Erinnerungen und Vorstellungsbildern ermöglicht ein stetes Anwachsen der Metaphern und damit ein immer breiteres Repertoire an möglichen Sinngebungen.

\section{Philosophie des Geistes: Kann es ein Unbewusstes geben?}

Als Zielvorstellung meiner Arbeit peile ich also eine Vorstellung von Triebbestimmtheit der Menschen an, gemäss derer letztere durch den Kontakt mit anderen hochgradig formbar sind und geistige Bedeutungen kulturell erwerben. Der Erwerb von Bedeutungen entspricht einer Sinnsuche, die durch die Notwendigkeit, andere zu verstehen entsteht. In der Konsequenz aber führen die Verschiebungen der Bedeutungen zu einem Prozess der ständigen Neuschöpfung und Ausbreitung von Metaphern, ikonischen Zeichen und Sprachbildern. Die wechselseitige Verbreitung und Hemmung dieser Bilder und Gedanken wird von biologischen Triebkräften angetrieben und mit diesen verwebt und verschränkt. Ich habe bereits angedeutet, dass Tomasello und Fonagy darin übereinstimmen, dass sie Bedeutungen externalistisch erklären.

Ich möchte im Folgenden einige Argumente aus einer angelsächsischen Diskussion zwischen Philosophie und Psychoanalyse anführen, die sich mit der Frage befassen, ob das Freud'sche Unbewusste mit einer externalistischen Bedeutungstheorie überhaupt vereinbar sei. In der angelsächsischen Philosophie des letzten Jahrhundertes drehten sich viele Auseinandersetzungen darum, wie unsere intuitiven Vorstellungen dessen, was wir tun, wenn wir denken, damit in Einklang zu bringen sind, dass wir an einen Geist, der sich in seinem Funktionieren grundsätzlich von der Materie unterscheidet, nicht mehr glauben (vgl. auch Bieri 1993). Es geht also um die Überwindung des Cartesianismus. In Bezug auf das Denken bedeutet das auch, dass wir uns von der Vorstellung, dass unser Wissen auf Erfahrungen der einzelnen Subjekte gründe und dass wir selber zu unserem eigenen Innenleben einen privilegierten Zugang hätten, der uns letztlich die übrige Welt erschliessen liesse, verabschieden. Die Vorstellung, dass die Bedeutungen von Aussen kommen, wird als Externalismus bezeichnet und kann mit dem amerikanischen Bedeutungstheoretiker Donald Davidson in Verbindung gebracht werden (vgl. Davidson 1989, Cavell 1997: 51 f.). Davidson fragt danach, wie Bedeutungen überhaupt möglich seien. Um den Hintergrund verständlich zu machen, auf dem sich die Argumente der hier folgenden Auseinandersetzungen bewegen, möchte ich die für uns hier wichtigen Antworten, die Davidson auf diese Frage gibt, stichwortartig anführen. Gemäss Davidson ist Verhalten und sind sprachliche Äusserungen immer nur bezogen auf bestimmte Situationen überhaupt ver- 
stehbar. Die sich in Kommunikation befindenden Gesprächspartner schreiben einander nachvollziehbare Intentionalitäten zu. Dasselbe gilt für Personen, die einander, ohne zu sprechen, beobachten. Wenn unterdeterminierte oder nicht eindeutige Äusserungen vorliegen (oder auf den ersten Blick nicht nachvollziehbare Handlungen beobachtet werden), wird der Gesprächspartner aufgrund des gemeinsamen Vorverständnisses der Situation, innerhalb derer die Äusserung geschieht, und aufgrund der Annahme, dass der andere sich intentional und rational verhalte, trotzdem verstanden. Die Grosszügigkeit, mit der Äusserungen und Handlungen von anderen ein Sinn zugeschrieben wird, nennt Davidson das Prinzip Charity. Weiter nimmt Davidson eine holistische Position ein. Gemeint ist damit, dass Davidson die ganzheitliche Situation, die die Gesprächsteilnehmer umfasst, in den Prozess der Verständigung mit einbezieht. Verständigung ist nur auf dem Hintergrund unzähliger, bereits feststehender Bedeutungen möglich. Davidson kommt den oben angeführten Gedanken zur Theory of Mind in seiner Formulierung der Triangulation nahe: Bedeutung ist nur möglich, wenn sich zwei Menschen zusammen auf etwas Drittes, die Realität beziehen. Mit der Triangulation (vgl. z. B. 1989 in 1993: 81 ff.) wird angesprochen, dass die Teilnehmer der Situation ihre Interpretationen über das Dritte vergleichen und sie glauben, dass sie die Wahrheit über dieselbe erkennen können. Man könnte auch sagen, sie glauben, dass sie recht haben, in dem, was sie behaupten. Es wäre interessant, das Davidson'sche Konzept der Triangulation mit demjenigen der Triangulierung, wie sie als psychologischer Effekt des Ödipuskomplexes von verschiedenen Richtungen der Psychoanalyse postuliert wird, noch näher zu vergleichen (vgl. dazu Cavell 1998). Davidson selbst hat sich in einem 1982 erschienen Artikel mit Freud auseinandergesetzt, ohne allerdings diese interessante Parallele anzusprechen. Sein Problem in der angesprochenen Arbeit, die, wie ich gleich ausführen werde, von Psychoanalytikern aufgenommen und hinterfragt worden ist, ist die Frage, wie das Freud'sche Unbewusste zu denken sei angesichts seiner Vorannahme, dass Verstehen und Bedeutung immer im Zusammenhang mit der Rationalitätserwartung der Teilnehmer gesehen werden muss. Unter dem Titel Paradoxes of Irrationality entwickelt Davidson eine recht eigenwillige Freud-Interpretation. Sie beinhaltet, wie Sebastian Gardner das später benennen wird, die Sub-System-Theorie. Davidson führt aus, dass die sich selbst widersprechende Selbstinterpretation möglich sei, weil die Wünsche und Meinungen eines Menschen nicht zwingend kohärent seien müssen. Hingegen würden «eine Reihe halb unabhängiger Strukturen» (2006: 287) im Geist eines Menschen existieren, die in einer bestimmten Hinsicht Personen ähnlich seien: Zusammenpassende Meinungen, Wünsche und Überzeugungen würden aufei- 
nander Bezug nehmen (ebd.). Die so entstehenden Sinngeflechte würden dann für sich genommen wieder rationale Elemente enthalten, auch wenn sie aus der Sicht eines andern Persönlichkeitsanteiles irreal wirken könnten (ebd.: $291 \mathrm{f}$.). Gemäss Davidson ist das Freud'sche Unbewusste, wenn man es denn auf diese Weise versteht, im Rahmen der von ihm vorgeschlagenen externalistischen Bedeutungstheorie denkbar. Diese Spaltung des Geistes, die für Davidson mit Freuds Aussagen über das Unbewusste übereinstimmen, könnte man vielleicht als vertikal beschreiben, während jene in Bewusst und Unbewusst, bei der man die von Freud mitformulierten Eigenschaften des Unbewussten mitdenkt, gemäss denen sich die beiden persönlichkeitsähnlichen Zentren bezüglich Rationalität stark unterscheiden, als horizontal imponiert. Marcia Cavell, die Partnerin von Davidson, die selbst Psychoanalytikerin ist, hat die Gedanken der analytischen Philosophie mit jenen der Psychoanalyse in verschiedenen Arbeiten aufeinander bezogen (1993, 1998, 2003). In The Psychoanalytic Mind führt sie aus, was das Davidson'sche Verständnis für die Psychoanalyse bedeutet:

«Einfühlung〉 [‘empathy»] kann keine Frage dessen sein, dass ich irgendwie aus meinem Geist hinaus in Ihren hereinkomme, sondern sie beruht vielmehr auf der Entdeckung und Ausweitung der gemeinsamen Basis, indem ich meine Vorstellungskraft hinsichtlich der Überzeugungen und Wünsche einsetze, bezüglich derer Ihr Verhalten Ihnen als mehr oder weniger vernünftig erscheint. ...» (1997: 58)

Hier wird also auch für den Prozess der Psychoanalyse die Externalität der Bedeutungen behauptet. Weiter unten spricht sie das Netz von Bedeutungen an, innerhalb dessen die Verständigung zwischen Analytiker und Patient möglich ist:

«Und das zweite ergibt sich aus dem ersten: dass nämlich der Psychoanalytiker und auch sein verrücktester Patient, wenn er überhaupt verstanden werden kann, viele Überzeugungen und Wünsche gemeinsam haben müssen. Von diesen als seiner Grundlage ausgehend, arbeitet der Analytiker darauf hin, auch diejenigen zu verstehen, die nicht gemeinsam und auf den ersten Blick vielleicht sogar zusammenhanglos sind.» (ebd. 59) 
Meiner Meinung nach könnte die Beschreibung der psychoanalytischen Situation, die Cavell vornimmt, für viele Psychoanalytiker heilsam sein. Der Tatsache, dass unsere Deutungen immer eingebettet sind in einen Rahmen von gemeinsamen Annahmen, die wir mit unseren Klienten teilen, wird nämlich viel zu wenig Beachtung geschenkt. Weiter erachte ich es für verdienstvoll, dass Alltagsdenken und psychoanalytische Vorstellungen vom Denken zusammengebracht werden, auch dies ein Desiderat innerhalb der psychoanalytischen Theoriebildung. Ich kann Cavell auch folgen, wenn sie herleitet, dass Freud auf den ersten Blick irrational anmutenden Symptomen mit seinen Deutungen einen neuen Deutungshintergrund verpasst, vor dem sie dann wiederum rational erscheinen.

Aber ich frage mich, ob diese Ergänzungen und Präzisierungen dessen, was in der psychoanalytischen Situation passiert, zwangsläufig zu einem vollständigen Aufgeben der Konzepte Primärprozess und Trieb führen müssen (vgl. Cavell 1993: $161 \mathrm{ff}$. und zusammenfassend Snelling 1995)! Ich werde deshalb zwei weitere philosophische Arbeiten vorstellen, die sich intensiv mit der Frage beschäftigen, ob und wie die psychoanalytischen Grundbegriffe zu retten sind. Die Arbeiten von Sebastian Gardner (1993) und Linda Brakel (2009) sind beides ernstzunehmende Auseinandersetzungen zwischen Philosophie und Psychoanalyse, und sie beziehen sich beide auf Davidson/Cavell, ja sind vielleicht sogar vom ketzerischen Versuch Cavells, einen Teil des Freud'schen Vokabulars einfach zu streichen, angeregt worden.

Gardners Argumente gegen die Abschaffung des Primärprozesses endet bei den Kleinianischen unbewussten Phantasien (vgl. auch Sturdee 1995), Brakel plädiert dafür, den Begriff des Irrationalen (der in der Titelgebung Gardners eindeutig die Bezugnahme auf die Davidson'sche Arbeit anzeigt) durch denjenigen des A-Rationalen zu ersetzen. Das Grundanliegen beider Arbeiten ist dasselbe: Sie möchten das psychoanalytische Konzept des Primärprozess nicht abschaffen, sondern ernster nehmen, als es normalerweise genommen wird. Seinen Status als Residuum von fehlgeleiteten und abgewehrten Denkprozessen soll es loswerden. Die Prinzipien, die Freud für den Primärprozess postuliert, sollen als Grundlagen des Denkens überhaupt gewürdigt werden, Grundlagen, die dem rationalen Denken vorangehen.

In ihrer Argumentation lässt sich Brakel, die praktizierende und forschende Analytikerin aus Michigan, stark auf die Denkfiguren der analytischen Philosophie ein. Sie argumentiert, dass zum Verständnis des Primärprozesses die Haltungen Überzeugungund Wunsch (Wunsch im Sinne von Handlungsziel; belief and desire), die für das Verstehen in der analytischen Philosophie als grundlegend angesehen 
werden, durch das dem Primärprozess entsprechende Paar Phantasie und Wunsch (Wunsch im Sinne von leerem Wunsch; phantasy and wish) ersetzt werden müssten. Sie kommt zum Schluss, dass die unbewusste Phantasie wohl etwas repräsentiere, aber keine Aussage, im Sinne einer Überzeugung mache und schliesslich findet sie auch ein Argument für die Existenz des Primärprozesses, das sich, ganz wie die modernen Argumente der Biologie, an der Evolutionstheorie orientiert.

All diese Argumente sollten für eine Evaluation natürlich im Einzelnen diskutiert und untersucht werden. Ich werde hier einen anderen Weg einschlagen. Die Frage, ob der Primärprozess phylogenetisch und ontogenetisch dem Sekundärprozess vorangehe, wie Freud angenommen hat und wie Gardner und Brakel sich zu verteidigen anschicken, oder ob er, wie die externalistische Bedeutungstheorie nahe legt, erst als Reaktion auf das, was Freud als Sekundärprozess bezeichnet, entsteht, quasi als dark side der Verständigung zwischen Menschen, werde ich vorerst noch offen lassen.

Ich möchte hier stattdessen nochmals auf das Konzept des Triebes zurückkommen. In der analytischen Philosophie des Geistes geht es u. a. darum, dass man sich Rechenschaft darüber geben möchte, wie der Übergang von Biologie zu dem, was im intuitiven Verständnis nach wie vor als Geist bezeichnet werden kann, konzipiert werden sollte. Und bekanntlich steht der Trieb gemäss Freud zwischen Biologie und Psyche, und ist deshalb geeignet, zu vermitteln.

Die Frage derVermittlung von Biologie und Geist wurde in der Philosophie unter anderem anhand der Intentionalität eingehend diskutiert. Der Begriff ist in die moderne Philosophie eingeführt worden von Franz Brentano (1874), der überdies auch als einer der Lehrer Freuds gilt. Brentano problematisiert mit dem Konzept der Intentionalität die Tatsache, dass wir im Geist ein Drittes, eine Sache haben (können). Allerdings war für Brentano - anders als für die modernen amerikanischen Philosophen, die den Begriff seit den 70er Jahren aufgenommen haben - die dualistische Sichtweise, die Materie und Geist als verschieden wirkende Prinzipien kennzeichnet, noch kein Problem. Vielmehr war für Brentano die Intentionalität gerade Ausdruck des geistigen Prinzips; was ihn jedoch beschäftigte, war, wie die Beziehung zwischen der im Aussen gedachten Realität zu diesen intentionalen, inneren Objekten zu denken sei.

Bei der amerikanischen Beschäftigung mit der Intentionalität stellt sich diese Frage etwas anders. Man ist bemüht, die Intentionalität des Denkens so zu formulieren, dass sie mit naturwissenschaftlichen Aussagen nicht in Konflikt kommt. Dieses Projekt nennt sich Naturalisierung der Intentionalität. Das heisst, es wird versucht, die Intentionalität des Denkens im Rahmen einer naturwissen- 
schaftlichen Sicht auf die Welt zu verstehen. Besonders prominent geworden ist in dieser Hinsicht die Philosophin Ruth Millikan, an deren Vorgehen sich Linda Brakel bei ihrer evolutionsbiologischen Begründung des Primärprozesses orientiert (vgl. Brakel 2009: $63 \mathrm{ff}$. ).

Ich hoffe, Sie nehmen es mir nicht übel, wenn ich an dieser Stelle auf mein Steckenpferd, meine Beschäftigung mit dem amerikanischen Philosophen Charles Sanders Peirce, zu sprechen komme. Peirce selber hat den Begriff der Intentionalität zwar nicht benutzt, seine Zeichentheorie ist indessen, wie zwei seiner modernen Interpreten hervorheben, für ein Studium derselben sehr geeignet. Stefan Kappner (2004a/2004b) zeigt dies interessanterweise ebenfalls anhand der Arbeit von Millikan, die er als teleosemantisch bezeichnet. Dabei verweist die Teleologie, die im Begriff steckt, auf die Zielgerichtetheit der Prozesse, hier auf ihre Funktion, die sie im Rahmen der Evolution zu erfüllen haben. Semantisch ist die Theorie deshalb, weil sie Bedeutungen erklären will. Zur näheren Bestimmung der Teleosemantik zitiere ich Kappner:

"Teleosemantische Theorien befassen sich auf dezidiert biologischer Grundlage mit dem Inhalt von Zeichen oder Repräsentationen. Ihr Ausgangspunkt ist ein Funktionsbegriff, der sich im Anschluss an teleologische (evolutionäre) Erklärungen in der Biologie gewinnen lässt und meist proper function [...] genannt wird. Als eigentliche Funktion einer Struktur, einer Eigenschaft oder eines Verhaltens soll diejenige Wirkung angesehen werden, die nach unseren evolutionären Hypothesen für die natürliche Selektion dieser Struktur, dieser Eigenschaft oder dieses Verhaltens verantwortlich ist. Mit Hilfe dieses Funktionsbegriffs ergibt sich eine normative Unterscheidung zwischen funktionierenden und nicht funktionierenden biologischen Einheiten, die sich allein mit Hilfe einer physiologisch-zergliedernden Beschreibung nicht gewinnen liesse.

[...] Wie die Identifikation einer eigentlichen Funktion zugleich die Möglichkeit eröffnet, Fehlfunktionen auszumachen, ergibt sich mit einer wie auch immer gerechtfertigten Zuordnung eines Inhalts zu einer Repräsentation oder einem Zeichen die Möglichkeit, von Fehlrepräsentationen zu sprechen, nämlich in solchen Fällen, in denen der Inhalt eines Zeichens entweder nicht existiert oder nicht so existiert, wie er repräsentiert wird.» (ders. 2004b: 162) 
Ich erlaube mir, die sehr knapp gefasste Charakterisierung des Projekts von Ruth Millikan durch Kappner auf noch einfachere Weise zu erklären (vgl. auch Millikan 1989). Sowohl in der Biologie wie auch in der Intentionalitätsforschung von Millikan wird davon ausgegangen, dass das zu untersuchende Phänomen, hier der Inhalt eines Gedankens, eine Funktion habe. Diese Funktion wird als eigentliche (proper) Funktion bezeichnet. Damit wird hervorgehoben, dass sie, die die Eigenschaft hat, die evolutionäre Fitness des betreffenden Individuums zu erhöhen, auch fehlgehen kann. Die Möglichkeit, auch falsche Gedanken erklären zu können, wird von jeder Theorie, die das Denken beschreibt, gefordert. Gemäss Ruth Millikans Verständnis der Evolutionstheorie erlaubt, ja erfordert diese biologische Theorie den Bezug auf Normen und Ziele. Nachdem sie ausgeführt hat, dass eine Reihe von Kollegen, die sich damit befassen, wie man geistige Inhalte herleiten könne, auf teleologische Theorien setzen, räumt sie ein:

"I fully agree, however, that an appeal to teleology, to function, is what is needed to fly a naturalist theory of content.» (ebd. 283)

Teleologische Theorien werden in der naturwissenschaftlichen Forschung normalerweise vermieden, und die Beschäftigung mit Normen und Zielen wird dem Bereich der Geisteswissenschaften zugeordnet. Millikans Naturalisierung der Intentionalität basiert auf der Annahme, die Evolutionstheorie, die in den biologischen Wissenschaften heute sehr verbreitet ist, sei eine naturwissenschaftliche Theorie. Mit ihrem Bezug auf Soll-Zustände weicht diese indessen von einem der Grundsätze, die man normalerweise für die Charakteristika naturwissenschaftlicher Theorien hält, erheblich ab.

In einem nächsten Schritt werde ich nun zeigen, dass sich auch bei Peirce eine Parteinahme für teleologische Theorien findet. Auch werde ich schildern, inwiefern die Beschäftigung mit Peirces Zeichentheorie für Forscher, die sich für die Intentionalität interessieren, sehr wichtig sein könnte.

Der zweite Peirce-Interpret, den ich nennen möchte, Thomas Short (2007), zeigt in seinen Ausführungen zu Peirces Zeichentheorie, dass dieser das Verhältnis zwischen Materie und Geist als kontinuierlich begreift. Die Frage des Dualismus (Materie-Geist) hatte ich oben bereits gestreift. Viele heutige Philosophen haben sich für den Materialismus entschieden, d. h. sie plädieren für eine Eliminierung des Geistes zugunsten der Materie - oder sie halten die Frage nach Geist und Materie für falsch gestellt. Für Peirce, der einer der ersten war, der die cartesianische Weltsicht kritisierte, war die Frage eminent wichtig und er hat die Kontinuität 
des Überganges betont. Dies zeigt sich einerseits darin, dass er, als Kenner der Naturwissenschaften seiner Zeit, der Meinung war, dass auch rein naturwissenschaftliche Methoden wie z.B. die Statistik und das zweite thermodynamische Gesetz finale Kausalitäten enthalten, wie Peirce dies nennt (ebd. 117-150). Mit finalen Kausalitäten ist angesprochen, dass das Ursache-Wirkung-Prinzip nicht immer nur in die eine Richtung wirke, sondern auch eine Umkehrung der Richtung denkbar sei (dass etwas vom Ziel, von der Norm her bestimmt ist).

Die Kontinuität zwischen Materie und Geist kommt bei Peirce auch da zum Ausdruck, wo er Zeichenprozesse im menschlichen Denken, im Kommunikationsverhalten, aber auch im Tierreich, ja sogar in biologischen Prozessen feststellt. Die allumfassende Präsenz von Zeichenprozessen führt bei Peirce tendenziell sogar dazu, dass es zuweilen schwierig wird, abzugrenzen, worin sich das menschliche Denken denn von den (übrigen?) biologischen Prozessen überhaupt noch unterscheidet. Thomas Schlicht hat in jüngerer Zeit vorgeschlagen, sich das Entstehen und Funktionieren der Intentionalität in Stufen (2008) vorzustellen. Das Stufenmodell der Intentionalität weist meiner Meinung nach in die richtige Richtung, obwohl Schlicht leider Peirce, für dessen Vorstellungen dieses Modell so viel Erhellung bringen könnte, nicht erwähnt. Es wäre auf jeden Fall reizvoll, Schlichts vier Stufen der Intentionalität, die dieser anhand der Kleinkindentwicklung (und anhand ganz ähnlicher Materialien wie Fonagy et al.) vorstellt, mit den Stufen des Funktionierens von tierischen und biologischen Zeichenprozessen zu ergänzen. Die vier von Schlicht vorgeschlagenen Stufen unterscheiden sich vor allem darin, wie stark sich die Zeichenprozesse auf sich selber beziehen können. So wird für das Kleinkind eine Art Reiz-Reaktion angenommen, die nach der 9-Monatsrevolution vom Lernen durch Nachahmung abgelöst wird. Auf der dritten Stufe wird dann die Einbildungskraft wichtig, das Kind kann als-ob-Situationen erfinden, verstehen und von authentischen Situationen unterscheiden. Schliesslich folgt auch bei Schlicht als vierte Stufe die Theory of Mind.

Ich würde den stufenweise sich entwickelnden Prozesses der Intentionalität als kontinuierlichen Aufstieg von biologischen Funktionen zu Denkoperationen und menschlicher Kommunikation verstehen. Dies bringt auch mit sich, dass in den höheren Stufen der Intentionalität die vorangehenden, tieferen Stufen (im Hegel'schen Sinne) aufgehoben sind, also immer mitgedacht werden müssen. Daraus ergibt sich die Forderung nach einer embodied theory of mind. Zur Illustration dieser Notwendigkeit werde ich nun Schlichts Charakterisierung einer der niederen Formen von Intentionalität zitieren: 
«Diese einfache Form von Intentionalität ergibt sich typischer Weise aufgrund von Repräsentationen eines Körper- oder Wahrnehmungszustandes und erfordert noch keinerlei Wissen des Organismus über sich selbst als handelndes Subjekt. So kann z.B. die Wahrnehmung eines roten runden Gegenstandes ein Baby dazu führen, nach dem roten Ball vor ihm zu greifen.» (ebd. 69/70, Hervorhebung des Autors)

Hier geht es um Erlebensformen, die der sozialen Interaktion, in der es propositionale Intentionalität gibt, vorangehen. Um jedoch die Verbindung und den Prozess der Entwicklung von biologischer Basis mit einfachen Formen der Intentionalität hin zu weiterführenden Formen der Intentionalität zu verstehen, brauchen wir Konzepte wie den Freud'schen Triebbegriff. Wenn ich an dieser Stelle also nochmals den Triebbegriff empfehle, möchte ich an dieser Stelle seine biologische Seite betonen: Der biologische Antrieb kann als grundlegender Motor für die Teleologie der Entwicklung, sowie später jene des Sprechens, Handelns und Denkens herangezogen werden. Wie oben bereits angeführt, wird die Variabilität des Objekts als die Einfallsstelle gesehen, an der eine externalistische Bedeutungstheorie angekoppelt werden kann. Der Primärprozess und der Sekundärprozess sind wie die zwei Seiten einer Medaille: Sie entwickeln sich gleichzeitig, beziehen sich aufeinander und grenzen sich voneinander ab. Die Spannung zwischen ihnen treibt die Entwicklung, die nie abgeschlossen ist, vorwärts. Mit Hilfe des Triebbegriffes kann - mit Peirce und Short gesprochen - die finale Kausalität im Handeln, Denken und Träumen der Menschen beschrieben werden, ohne dass deswegen der persönliche Weg des Einzelnen durch normative Entwicklungsziele bereits festgelegt werden muss.

\section{Literatur}

Bieri, Peter (1993): Generelle Einführung. In Ders. (Hrsg.): Analytische Philosophie des Geistes. Bodenheim: Athenäum Hain Hanstein. 1-28.

Brakel, Linda (2009): Philosophy, Psychoanalysis, and the A-Rational Mind. New York: Oxford University Press.

Brentano, Franz (1874/ 1974): Psychologie vom empirischen Standpunkt. Hamburg: Meiner.

Britton, Ronald, Michael Feldmann und Edna O'Shaughnessy (1989/1998): The Oedipus Complex Today: Clinical Implications. London: Karnac Books. Deutsch: Der Ödipuskomplex in der Schule Melanie Kleins. Stuttgart: Klett-Cotta. 
Cavell, Marcia (1993/1997): The Psychoanalytic Mind. From Freud to Philosophy. Cambridge, MA and London; Deutsch: Freud und die analytische Philosophie des Geistes. Überlegungen zu einer psychoanalytischen Semantik. Stuttgart: Klett-Cotta.

Cavell, Marcia (1998): Triangulation, One's Own Mind and Objectivity. In Journal of Psycho-Analysis 79:449-466.

Cavell, Marcia (2003): The Social Character of Thinking. In Journal of the American Psychoanlytical Association 51: 803-824.

Davidson, Donald (1989/1993): Epistemology Externalized. Unveröffentlichtes Manuskript. Deutsch: Externalisierte Bedeutungstheorie. In Der Mythos des Subjektiven. 65-83.

Davidson, Donald (1982/2006): Paradoxes of Irrationality. In Richard Wollheim und James Hopkins (eds): Philosophical Essays on Freud. New York and Cambridge: Cambridge University Press. Deutsch: Paradoxien der Irrationalität. In Donald Davidson: Probleme mit der Rationalität. Frankfurt am Main: Suhrkamp. 285-315.

Davidson, Donald (1993): Der Mythos des Subjektiven. Philosophische Essays. Ditzingen: Reclam.

Fonagy, Peter et al. (2002/2006): Affect Regulation, Mentalization and the Development of the Self. New York: Other Press. Deutsch: Affektregulation, Mentalisierung und die Entstehung des Selbst. Stuttgart: Klett-Cotta.

Freud, Sigmund (1915a/1982): Triebe und Triebschicksale. In Studienausgabe Band III. Frankfurt am Main: Fischer Wissenschaft. 75-102.

Gardner, Sebastian (1993): Irrationality and the Philosophy of Psychoanalysis. Cambridge: Cambridge University Press.

Hobson, Peter (2002/2003): The Cradle of Thought. Exploring the Origins of Thinking. London: Macmillan. Deutsch: Wie wir denken lernen. Gehirnentwicklung und die Rolle der Gefühle. Düsseldorf und Zürich: Walter Verlag.

Kappner, Stefan (2004a): Intentionalität aus semiotischer Sicht. Peirceanische Perspektiven. Berlin, New York: Walter de Gruyter.

Kappner, Stefan (2004b): Der Inhalt innerer Repräsentationen im Licht von Peirces Zeichenbegriff. In Johann C. Marek und Maria E. Reicher (Hg): Erfahrung und Analyse. Beiträge des 27. Internationalen Wittgenstein Symposiums. Kirchberg am Wechsel. 162-164.

Millikan, Ruth Garrett (1989): Biosemantics. In The Journal of Philosophy, 86: 6. 281-297. 
Schlicht, Tobias (2008): Ein Stufenmodell der Intentionalität. In Patrick Spät (Hg): Die Zukunft der Philosophie des Geistes. Paderborn: Mentis. 59-91.

Schore, Allan N. (1994): Affect Regulation and the Origin of the Self: The Neurobiology of Emotional Development. Hillsdale N.J.: Erlbaum Associates.

Short, Thomas. L. (2007): Peirce's Theory of Signs. Cambridge et al.: Cambridge University Press.

Snelling, David (1995): Marcia Cavell: The Psychoanalytic Mind. In Mind 104: 415: 892-896.

Stern, Daniel (1985/1993): The Interpersonal World of the Infant: A View from Psychoanalysis and Developmental Psychology. New York: Basic Books. Deutsch: Die Lebenserfahrung des Säuglings. Stuttgart: Klett-Cotta.

Sturdee, P.G. (1995): Irrationality and the Dynamic Unconscious: The Case for Wishful Thinking. In Philosophy, Psychiatry and Psychology 2: 2: 165-174.

Tomasello, Michael (1999/2002): The Cultural Origins of Human Cognition. Cambridge, MA und London, UK. Deutsch: Die kulturelle Entwicklung des menschlichen Denkens. Frankfurt am Main: Suhrkamp.

\section{Anmerkung}

1 Vgl. Stern 1985, in neuerer Zeit auch neurophysiologisch: Hobson 2002 und Schore 1994. 